\title{
Many Body Theory of Charge Transfer in Hyperthermal Atomic Scattering
}

\author{
J. B. Marston \\ Department of Physics \\ Box 1843 \\ Brown University \\ Providence, RI 02912 U.S.A. \\ D. R. Andersson \\ E. R. Behringer \\ B. H. Cooper \\ C. A. DiRubio \\ G. A. Kimmel \\ and \\ C. Richardson \\ Laboratory of Atomic and Solid State Physics \\ Cornell University \\ Ithaca, NY 14853-2501 U.S.A.
}


ABSTRACT: We use the Newns-Anderson Hamiltonian to describe many-body electronic processes that occur when hyperthermal alkali atoms scatter off metallic surfaces. Following Brako and Newns, we expand the electronic many-body wavefunction in the number of particle-hole pairs (we keep terms up to and including a single particle-hole pair). We extend their earlier work by including level crossings, excited neutrals and negative ions. The full set of equations of motion are integrated numerically, without further approximations, to obtain the many-body amplitudes as a function of time. The velocity and work-function dependence of final state quantities such as the distribution of ion charges and excited atomic occupancies are compared with experiment. In particular, experiments that scatter alkali ions off clean $\mathrm{Cu}(001)$ surfaces in the energy range 5 to 1600 $\mathrm{eV}$ constrain the theory quantitatively. The neutralization probability of $\mathrm{Na}^{+}$ions shows a minimum at intermediate velocity in agreement with the theory. This behavior contrasts with that of $\mathrm{K}^{+}$, which shows virtually no neutralization, and with $\mathrm{Li}^{+}$, which exhibits a monotonically increasing neutral fraction with decreasing velocity. Particle-hole excitations are left behind in the metal during a fraction of the collision events; this dissipated energy is predicted to be quite small (on the order of tenths of an electron volt). Indeed, classical trajectory simulations of the surface dynamics account well for the observed energy loss, and thus provide some justification for our truncation of the equations of motion at the single particle-hole pair level. $\mathrm{Li}^{+}$scattering experiments off low work-function surfaces provide qualitative information on the importance of many-body effects. At sufficiently low work function, the negative ions predicted to occur are in fact observed. Excited neutral Li atoms (observed via the optical $2 \mathrm{p} \rightarrow 2 \mathrm{~s}$ transition) also emerge from the collision. A peak in the calculated $\operatorname{Li}(2 \mathrm{p}) \rightarrow \operatorname{Li}(2 \mathrm{~s})$ photon intensity occurs at intermediate work function in accordance with measurements.

PACS: 34.70.+e, 79.20.Rf, 79.80.+w, 71.10.+x 


\section{INTRODUCTION}

The single-particle picture of resonant charge transfer, based on a time-dependent NewnsAnderson Hamiltonian, successfully explains the observed work-function dependence of the neutralization probability of positive hyperthermal alkali ions that sputter ${ }^{1}$ or scatter ${ }^{2,3}$ off metallic

surfaces. (For a review, see Ref[4].) The key simplifying feature of this approximation is the absence of multiple atomic degrees of freedom: the electrons are treated as spinless Fermions that either occupy or do not occupy a single valence orbital of the alkali ion. (The Pauli exclusion principle guarantees that double occupancy cannot occur.) Analytical solutions to the single-particle problem can be obtained. ${ }^{5}$

Yet questions remain. When the atomic orbital is degenerate, the single-electron picture breaks down. For example, the valence s-orbital of a positive alkali ion may be filled with either a spin-up or a spin-down electron. The degeneracy is a non-trivial complication, because strong correlations must exist. In the alkali case, once a spin-up electron transfers to the ion, subsequent attempts to transfer a spin-down electron are discouraged by the strong intra-atomic Coulomb repulsion between the two valence electrons. (The repulsion manifests itself in the fact that the electron affinity energy of alkali atoms is much smaller than the ionization energy.) The complication is reminiscent of the Kondo problem of a magnetic ion embedded in a metal where the spin residing on the impurity couples to the conduction electrons via virtual processes which allow a second electron to temporarily jump onto the ion at some large energy cost. The Kondo effect is a collective phenomenon characterized by strong many-body correlations induced by the impurity spins. Thus the fact that real electrons come in two forms (spin up or down) means that the single-particle picture really does not describe even the simplest problem of a single atomic orbital. It is therefore interesting to inquire into why the single-particle results fit the neutralization experiments so well.

Multiple atomic orbitals are another source of degeneracy and correlations. For example, the affinity p-orbitals of a neutral oxygen atom are degenerate, at least when the atom is far from the metal surface. When one of these orbitals acquires an electron, further transfers (which would 
yield an $\mathrm{O}^{--}$ion) are energetically disfavored. Langreth and Nordlander recently emphasized ${ }^{6}$ that the neglect of such correlations can lead to qualitatively incorrect results. For example, the $\mathrm{p}_{x}$ and $\mathrm{p}_{y}$ orbitals of an oxygen atom couple only weakly to a metal surface with its normal in the $\mathbf{z}$ direction. Therefore the $\mathrm{p}_{z}$ orbital should fill first as the atom approaches the surface. Once filled, additional electrons will be locked out of the $\mathrm{p}_{x}$ and $\mathrm{p}_{y}$ orbitals by the Coulomb repulsion. As the atom departs from the surface, there will be ample time, if the atom is not traveling too fast, for the $\mathrm{p}_{z}$ orbital to empty, yielding a neutral oxygen as the final state. Had the intra-atomic Coulomb energy been ignored, the final state would have been a negative ion, because the $\mathrm{p}_{x}$ and $\mathrm{p}_{y}$ orbitals would also fill when the atom is close to the metal and then retain their electrons as the atom moves away.

The problem resembles the much-discussed "Coulomb Blockade" ${ }^{7}$ which encumbers electrons that hop on to a small conducting dot of capacitance $C$. In the present case, the atom functions as a capacitor because extra energy is required to add a second electron.

To treat these many-body correlations, we resort to an approximate solution of the NewnsAnderson problem. We employ a systematic $1 / N$ expansion $(N$ is the spin degeneracy of the electrons and equals two for the physical case of spin up and down species) to study the dynamics of charge transfer involving multiple orbitals. The expansion is equivalent to a variational expansion of the many-body wavefunction in the number of particle-hole pairs. It was employed with success in the Kondo problem ${ }^{8}$ (the perturbation series converge rapidly when $\mathrm{N}$ is large enough). Indeed, the $1 / N$ expansion behaves qualitatively the same as the exact Bethe-ansatz solution. ${ }^{9}$ Brako and Newns ${ }^{10}$ applied it to the dynamical charge transfer problem in 1985 . We go further by including level crossings, excited atomic states, and affinity levels in the calculation. We find that the results closely match those of the single-particle picture over a broad range of parameters. Apparently, the single-particle picture works so well because the incorporation of spin and higher energy atomic states has little effect on the neutralization probability. On the other hand, the production of negative ions and excited neutrals becomes significant at low work functions. For these cases, the 
more complete theory is essential for a proper description of the observable physics.

The basic idea behind the $1 / N$ expansion is as follows: when $N$ is large, the amplitude for each of the $N$ types of electrons to transfer to or from the atom must be scaled back so that the overall charge-transfer rate for any of the $\mathrm{N}$ types of electrons stays reasonable. In this limit, the rate of formation of particle-hole pairs becomes smaller and smaller because these excitations are produced by processes in which an electron of a given species performs not one but two hops: once from a filled state in the metal to the atom and then another hop back to the metal into a state above the Fermi level. Particle-hole pair formation therefore becomes negligible in the $N \rightarrow \infty$ limit, and the many-body equations are simple. The advantages of this systematic solution of the many-body problem are two-fold: First, it is straightforward to identify the correction terms that appear at each order in the $1 / \mathrm{N}$ expansion. Second, we can test whether the $1 / \mathrm{N}$ expansion breaks down as $\mathrm{N}$ decreases from infinity down to the physical value of 2 (see below).

The present work is similar in some respects to calculations by Sulston and collaborators. ${ }^{11}$ These earlier calculations, however, included neither particle-hole excitations nor excited atomic states in the variational wavefunction. Later calculations by the same group incorporated these states $^{12}$ but all of the calculations employed a "local time approximation" of untested accuracy to simplify the equations of motion. This approximation alters the normalization of the many-body wavefunction which consequently has to be renormalized periodically during the integration forward in time. We avoid approximations of this sort by directly integrating the full set of equations of motion. We show below that particle-hole pairs play a crucial role in erasing memory of the initial state of the incoming atom; thus it is important to include them. The inclusion of particle-hole pairs also enables us to estimate the amount of energy dissipated by their formation. It is therefore possible to check, both theoretically and experimentally, the size of the errors attending the $1 / \mathrm{N}$ expansion since the single particle-hole channels represent corrections to the lowest-order $(N \rightarrow \infty)$ solution. Finally, by adding excited atomic states, we are able to make additional contact with experiment (which can detect optical transitions as the excited states decay). Competition between 
negative and excited neutral final states is important and explains newly obtained experimental data.

Many features of the theory can be tested experimentally. The most important unknowns are the set of distance-dependent couplings between the atomic states and the metal. We use recent first-principles calculations (in a single-particle approximation) of the couplings ${ }^{13,14}$. Scattering experiments off clean surfaces, by avoiding the complicated local variations in the electrostatic potential produced by adsorbates, yield quantitative information that check the validity of these parameters. Indeed, different alkali species ( $\mathrm{Li}, \mathrm{Na}$, and $\mathrm{K}$ ) exhibit qualitatively different behavior and the theory must account for these differences. Also, measurements of negative ion fractions and excited neutral yields (which become significant at relatively high velocities and low work functions) directly test the many-body features of the theory. Finally, experiments that measure energy dissipation during the collision process, combined with classical trajectory simulations, provide upper bounds on the amount of particle-hole excitations left behind in the metal. These bounds can be compared to the predicted losses due to electronic mechanisms. No one experiment is sufficient to establish the credibility of a theory with several parameters; rather a combination of tests is required. We make preliminary comparisons between our theory and several experiments below.

In section (II) we discuss a generalized Newns-Anderson Hamiltonian for resonant chargetransfer that includes level crossings, electron spin, excited neutrals, and negative ion states. The model serves as a starting point for extensions to more complicated situations that will be the focus of future work. The systematic solution of the many-body dynamics is presented in section (III). Section (IV) is devoted to a preliminary experimental evaluation of the theory. We address neutralization rates, the formation of excited neutrals and negative alkali ions off low work function surfaces, and the energy loss due to the formation of particle-hole pairs. Conclusions and a discussion of open questions are presented in section (V). 


\section{THE GENERALIZED NEWNS-ANDERSON MODEL}

To begin, we make several simplifying assumptions. We employ the Newns-Anderson Hamiltonian, ignore radiative and Auger charge transfer processes, and consider only resonant charge transfer. Charge transfer that involves the emission of a photon is suppressed relative to resonant charge transfer by a factor of $\alpha \approx 1 / 137$ (the fine-structure constant) and the inclusion of Auger processes is something we plan to address in future work. The electrons in the target metal are modeled as non-interacting spinning Fermions, albeit with renormalized parameters such as effective mass. Finally, the atom is modeled as a system with a finite number of discrete states moving along a fixed classical trajectory given by $z(t)$ where $z$ is the distance from the atom to the metal surface. (For a jellium model of the metal electrons, $z$ is the distance from the nucleus of the hyperthermal atom to the jellium edge.) Each of these states couples to the metal electrons when the atom is sufficiently close to the metal surface. Feedback between the electronic degrees of freedom and the trajectory is ignored in the formulation. This approximation should be adequate as long as the kinetic energy of the ion is much larger than the electronic energies.

The model is defined by the following generalized time-dependent Newns-Anderson Hamiltonian:

$$
\begin{aligned}
H(t) & =\sum_{a}\left[\epsilon_{a}^{(1)}(z) P_{1}+\epsilon_{a}^{(2)}(z) P_{2}\right] c_{a}^{\dagger \sigma} c_{a \sigma}+\sum_{k} \epsilon_{k} c_{k}^{\dagger \sigma} c_{k \sigma} \\
& +N^{-1 / 2} \sum_{a ; k}\left\{\left[V_{a ; k}^{(1)}(z) P_{1}+V_{a ; k}^{(2)}(z) P_{2}\right] c_{a}^{\dagger \sigma} c_{k \sigma}+H . c\right\} \\
& +\sum_{a>b} U_{a b} n_{a} n_{b}+\frac{1}{2} \sum_{a} U_{a a} n_{a}\left(n_{a}-1\right)
\end{aligned}
$$

Here $c_{a}^{\dagger \sigma}$ creates a spin $\sigma$ electron in orbital $a$ of the atom (ie. for Li, a $=0$ for the 2 s orbital, a $=1$, 2 , and 3 for $2 \mathrm{p}_{x}, 2 \mathrm{p}_{y}$, and $2 \mathrm{p}_{z}$, etc.) Likewise, $c_{k}^{\dagger \sigma}$ creates an electron of momentum $k$ and energy $\epsilon_{k}$ in the metal. Of course, $k$ is really a three-vector, but it may be regarded as a scalar without loss of generality by absorbing the three-dimensional aspects of the problem into $\epsilon_{k}$ and $V_{a ; k}$. We introduce the operators $P_{1}$ and $P_{2}$ to project respectively onto atoms with one or two valence electrons. These projectors, which may be written in terms of the orbital occupancies $n_{a} \equiv c_{a}^{\dagger \sigma} c_{a \sigma}$,

permit one to assign different orbital energies $\left(\epsilon_{a}^{(1)}\right.$ and $\left.\epsilon_{a}^{(2)}\right)$ and metal-atom couplings $\left(V_{a ; k}^{(1)}\right.$ and 
$\left.V_{a ; k}^{(2)}\right)$ to the two cases of neutral atoms and negative ions. An implicit sum over repeated upper and lower Greek indices is adopted; for now $N=2$ and $\sigma=1,2$ to represent the physical $\mathrm{SU}(2)$ case of spin up and down electrons. Actually, when $N>2$ additional projectors $P_{3}, P_{4}$, etc. should be included to account for the possibility of having, say, three SU(4) Fermions in the same orbital. Instead, we implicitly assume that these states have infinite energy and simply remove them from the Hilbert space. The removal of course has no effect on the physical $\mathrm{SU}(2)$ results, but is just a formal trick to keep the $1 / N$ expansion as simple as possible. For convenience, we also multiply the atom-metal coupling by a factor of $N^{-1 / 2}$. This factor allows one to take the $N \rightarrow \infty$ limit without rescaling $V_{a ; k}$. Finally, we neglect the possibility of spin-flip processes in our Hamiltonian: $H$ is invariant under global $\mathrm{SU}(2)$ [or more generally $\mathrm{SU}(\mathrm{N})]$ spin rotations.

$U_{a b}$ is the Coulomb repulsion between two electrons in valence shells $a$ and $b$ which in principle depends on $z$ but which in practice we assume to be constant. (The assumption can be relaxed if necessary.) As it stands, excited states of negative ions are permitted. But, because these high energy states are not expected to play a significant role in the many-body wavefunction, we eliminate them by taking $U_{a b} \rightarrow \infty$ when orbitals $a$ and $b$ are not the lowest s-orbital of the alkali atom.

We retain non-trivial time dependence in the orbital energies and atom-metal couplings of the model. The time-dependence enters through the ion trajectory, which we sometimes model as:

$$
\begin{aligned}
z(t) & =z_{f}-u_{i} * t ; t \leq t_{\text {turn }} \equiv\left(z_{f}-z_{0}\right) / u_{i} \\
& =z_{0}+u_{f} *\left(t-t_{\text {turn }}\right) ; t>t_{\text {turn }}
\end{aligned}
$$

Thus the trajectory starts at a distance $z_{f}$ far from the surface at time $t=0$. We account roughly for a decrease in the ion kinetic energy (due principally to the recoil of surface atoms during impact) and the change in the scattering angle here by instantaneously changing the initial perpendicular component of the ion velocity, $u_{i}$, to $u_{f}$ at the point of closest approach, $z_{0}$. (Another possible trajectory, discussed below, neglects the inward bound portion of the trip and instead starts the atomic motion headed in an outward direction starting from the point of closest approach.) More 
complicated time-dependent trajectories can be incorporated as needed. Attention must also be paid to the dependence of the atom-metal couplings and the effective density of states on the parallel component of the ion velocity. Note that the electronic states in the metal are shifted in momentum in the reference frame of the ion ${ }^{15}$. For now we ignore parallel velocity; the inclusion of this effect will be part of future refinements.

We define the Fermi energy to be zero and relate all other energies to it; the vacuum level lies above it at energy $\Phi$, the work function. Because of the image potential, the ionization levels of the atom $\epsilon_{a}^{(1)}$ shift upward as it approaches the metal surface:

$$
\epsilon_{a}^{(1)}(z)=\epsilon_{a}(\infty)+\Phi+e^{2} / 4 z
$$

Here $\epsilon_{a}(\infty)$ is the ionization energy of orbital $a$ of an isolated atom, which is taken to be a negative number. A more realistic model has the image shift saturate when the atom gets close to the surface. We account for the saturation by introducing a cutoff, $v_{\max }$, in the image potential. Also, the image plane does not coincide exactly with the metal edge; rather it can lie within a small distance of it. Therefore we introduce an adjustable parameter, $z_{i m}$, the distance from the surface at which the image saturates to value $v_{\max }$. So a better form for the ionization energy is given by:

$$
\begin{aligned}
\epsilon_{a}^{(1)}(z) & =\epsilon_{a}(\infty)+\Phi+\left(1 / v_{\max }^{2}+16\left(z-z_{i m}\right)^{2} / e^{4}\right)^{-1 / 2} ; z>z_{i m} \\
& =\epsilon_{a}(\infty)+\Phi+v_{\max } ; z<z_{i m} .
\end{aligned}
$$

The two parameters in Eq. [2.4] can to some extent be determined experimentally from an analysis of the ion trajectories and energies ${ }^{16}$. We typically take $v_{\max }=2.6 \mathrm{eV}$ and $z_{i m}=0.0 \AA$ for the $\mathrm{Cu}(001)$ surface. Especially interesting situations arise when the shift is large enough to push the ground state ionization energy above the Fermi energy at some crossing distance $z_{c}>z_{0}$. In these cases, neutralization probabilities can increase from nearly $0 \%$ to $100 \%$ as the velocity of outgoing positive alkali ion decreases. ${ }^{1,3}$ Local adsorbate induced electrostatic potentials are obviously not included in Eq. [2.4]. Since adding adsorbates to the surface is a convenient way of changing the work function, it is often necessary to consider local variations in the potential when fitting 
experimental results to theory ${ }^{17,3}$. We propose to compute averages over different trajectories as part of future work.

In contrast to the ionization levels, the affinity levels shift downward by $e^{2} / 4 z$ as the atom approaches the surface. In other words, the energy required to remove the two valence electrons bound to a negative ion (thereby making it a positive ion) is unaffected by the image charges. Thus, it is simply:

$$
\epsilon_{a}^{(2)}(z)=\epsilon_{a}(\infty)+\Phi
$$

The intra-atomic Coulomb repulsion between two electrons in the lowest s-orbital $(a=0)$ is then given by $U=A-\epsilon_{0}^{(2)}(\infty)=A-\epsilon_{0}(\infty)$ where $U \equiv U_{00}$ and $A$ is the electron affinity (also defined here to be negative).

The atom-metal couplings decay exponentially with distance when the atom is far from the metal surface because the atomic wavefunctions drop off exponentially with increasing distance from the atom and the electronic wavefunctions in the metal fall off exponentially with increasing $z$. Closer in, the couplings deviate from the pure exponential form. A systematic Laurent expansion of the logarithm of the couplings (we suppress the occupancy superscript) yields:

$$
V_{a ; k}(z)=\tilde{V}_{a ; k} \exp \left[a_{-1}(a ; k) / z+a_{1}(a ; k) z\right]
$$

[Note that $V_{a ; k}(z)$ need not be purely real. Nevertheless, we take it to be real in the following calculations.] Non-zero (and negative) $a_{-1}$ incorporates saturation in the growth of the coupling at short distances. Further terms $a_{-2}, a_{-3}$, etc. may be added to the Laurent expansion as needed. In the following calculations we generally ignore the $k$ dependence of the metal-atom coupling. This approximation is really quite severe. It is justified in so far as most of the resonant electronic processes occur close to the Fermi surface and the wavevector dependence of the couplings is smooth. Making this assumption for the singly-occupied orbitals,

$$
V_{a ; k}^{(1)}(z)=\tilde{V} \exp \left[a_{-1}(a) / z+a_{1}(a) z\right]
$$


we find that the functional form of Eq. [2.7] fits quite well values for $V^{(1)}(z)$ obtained from the single-particle widths calculated in Ref[13] without recourse to additional terms in the Laurent expansion.

At far to moderate distances, we expect the overlap $V_{0}^{(2)}$ between the metal states and the affinity orbital to be considerably larger than the overlap between the metal and the neutral ground state orbital because negative ions are very large in size. Previous models (for example, those of $\operatorname{Ref}[12], \operatorname{Ref}[18]$ and $\operatorname{Ref}[19])$ did not account for this difference: the same couplings were used for the affinity and ground state orbitals. In fact the rms radius of the $\mathrm{Li}^{-}$ion, calculated in a Monte-Carlo approach ${ }^{20}$, exceeds $2.0 \AA$. First-principle calculations of these couplings are of course desirable and we make use of recent computations by $\operatorname{Nordlander}^{14}$ (in a single-particle approximation) of the coupling between the metal and negative ions. Whether the single-particle approximation itself is adequate for the calculation of these couplings is a question that requires further investigation. 


\section{A SYSTEMATIC SOLUTION}

Before embarking on the systematic solution to the many-body Newns-Anderson system, we make some observations about other theoretical approaches. First it is clear that simply ignoring the intra-atomic Coulomb energy in the physical problem with electrons of up and down spins would give completely incorrect answers, even for the case of a single atomic orbital. For example, when a slow alkali ion bounces off a surface with work-function $\Phi$ which is less than the magnitude of the ionization energy, it should emerge neutralized: an electron will always be able to transfer from the metal to the valence orbital. Under these conditions, neglecting $U$ would mean that the atom actually emerges as a negative ion because if a spin-down electron hops from the metal to the atom, so will a spin-up electron, filling the orbital. One might think that the intra-atomic repulsion could be treated adequately in the Hartree-Fock approximation. But here again it is impossible to get neutral fractions greater than $50 \%$ because the two spin species remain uncorrelated ${ }^{18}$. In other words, when the neutral fraction becomes significant, so will negative ion formation. This situation is at odds with experiments that find nearly $100 \%$ neutral fractions.

Exact diagonalization of a Newns-Anderson Hamiltonian for targets consisting of just three atoms in a chain is relatively straightforward ${ }^{21}$. But because the Hilbert space becomes unmanageably large for more than a few atoms, and because the existence of a continuum of metal states is required for an adequate description of resonant charge-transfer, this method cannot be applied to the macroscopic metal targets that are of interest here. Nevertheless, it was found that the single-particle approximation describes the three-atom cluster reasonably well when the ionization and affinity energies of the atoms are very different ${ }^{21}$. This result anticipates our observation that both the single-particle and many-body pictures yield similar values for the alkali neutralization probability when the affinity levels lie well above the Fermi energy.

Successes in understanding the Kondo problem (the static limit of the Newns-Anderson Hamiltonian) suggest some different approaches. The slave-Boson Green's function method is a convenient technique for enforcing the constraint of single orbital occupancy in the $U \rightarrow \infty$ limit, and Langreth 
and Nordlander apply it to the resonant charge-transfer problem ${ }^{6}$. In the limit of low ion velocity and high temperature $[\Gamma(z) \beta<<2 \pi$ and $\alpha u<<2 \pi$ where $\beta$ is the inverse temperature, $u$ is the velocity in atomic units, and the width of the atomic levels is assumed to drop off exponentially: $\Gamma(z) \propto \exp (-\alpha z)]$ they obtain simple coupled master equations from the low-order equations for the occupancies of the atomic orbitals. The problem of finite intra-atomic Coulomb interactions may also be treated by extensions of this approximate method ${ }^{22}$. Unfortunately, the master equations are not justified at higher velocities; instead cumbersome Dyson equations must be solved.

Since we are primarily interested in the case of higher ion velocities (which enhance nonadiabatic survival of excited neutrals and negative ions) we prefer to follow the different, but related, systematic approach of Brako and Newns ${ }^{10}$ and group the full many-body electronic wavefunction into sectors containing more and more numbers of particle-hole excitations in the metal. Upon truncating the wavefunction at a given number of particle-hole pairs, we obtain a variational wavefunction that spans only a tiny portion of the entire Hilbert space. However, as long as the amplitude for the formation of particle-hole pairs during the ion-surface collision remains relatively small, we may view the wavefunction as a good approximation to the full one. (The expansion bears some resemblance to the "equations of motion method" employed by Kasai and Okiji ${ }^{19}$ and the coupled-cluster expansion of Sebastian ${ }^{18}$.) The amplitude for particle-hole pair production may be controlled at least formally by generalizing the two types of SU(2) electrons (spin up and down) to $\mathrm{N}$ types of $\mathrm{SU}(\mathrm{N})$ Fermions. Thus the spin index $\sigma$ now runs from 1 to $\mathrm{N}$. We show below that the amplitudes for terms involving more and more particle-hole pairs are reduced by higher and higher powers of $1 / \mathrm{N}$. As long as $\mathrm{N}$ is large enough, the errors introduced by the truncation of the Hilbert space should be small. We present theoretical and experimental evidence to show that even in the physical case $N=2$ higher-order terms in the expansion are small.

To begin, we decompose the many-body wavefunction into four sectors plus the remaining 
Hilbert space:

$$
\begin{aligned}
|\Psi(t)\rangle & =f(t)|0\rangle+\sum_{a ; k<k_{f}} b_{a ; k}(t)|a ; k\rangle+\sum_{k<k_{f}, l>k_{f}} e_{l, k}(t)|l, k\rangle+\sum_{q<k<k_{f}} d_{k, q}(t)|k, q\rangle \\
& + \text { (rest of Hilbert space) } .
\end{aligned}
$$

Each sector is a global $\mathrm{SU}(\mathrm{N})$ singlet. Non-singlet sectors can be ignored in so far as the initial state of the system (a closed shell positive alkali ion far away from an unperturbed, non-magnetic metal) and the Hamiltonian are both $\mathrm{SU}(\mathrm{N})$ singlets. Here the orthonormal basis states in different sectors of the Hilbert space are given by:

$$
\begin{aligned}
|a ; k\rangle & \equiv N^{-1 / 2} c_{a}^{\dagger \sigma} c_{k \sigma}|0\rangle \\
|l, k\rangle & \equiv N^{-1 / 2} c_{l}^{\dagger \sigma} c_{k \sigma}|0\rangle \\
|k, q\rangle & \equiv[N(N-1)]^{-1 / 2} c_{0}^{\dagger \alpha} c_{k \alpha} c_{0}^{\dagger \beta} c_{q \beta}|0\rangle .
\end{aligned}
$$

The reference state $|0\rangle$ represents a positive alkali ion (ie. an empty valence shell) along with the non-interacting Fermi-liquid at zero-temperature in the absence of any particle-hole excitations. The limits on the momenta ranges appearing in Eq. [3.1] are shorthand notation for $\epsilon_{q}<\epsilon_{k}<\epsilon_{f}$ and $\epsilon_{l}>\epsilon_{f}$ where $\epsilon_{f} \equiv 0$ is the Fermi energy. In other words, $k$ and $q$ label hole momenta, and $l$ labels particle momentum, so while $|l, q\rangle$ is a positive ion plus a particle-hole pair, the state $|k, q\rangle$ instead represents a negative ion with two holes in the metal. A schematic of the different sectors of the Hilbert space is presented in Figure [1]. Note that excited negative ions do not appear in Eq. [3.2]. These states are removed from the Hilbert space by hand since (as discussed above) we set $U_{a b} \rightarrow \infty$ for $a, b \neq 0$. We show below that terms involving two or more particle-hole pairs constitute higher-order corrections dropped in the approximate solution.

The time-dependent coefficients appearing in the many-body wavefunction Eq. [3.1] are amplitudes for the following states:

(1) $f(t)$ - A positive ion with no excitations in the metal. Note that $f(t=-\infty)=1$ describes the initial state of an experiment which directs incoming positive ions against the metal target.

(2) $b_{a ; k}(t)$ - A neutral atom with orbital $a$ occupied and a hole left behind in the metal at momentum $k$. 
(3) $e_{l, q}(t)$ - A positive ion and a single particle-hole pair in the metal (the electron has momentum $l$ and the hole has momentum $q$ ).

(4) $d_{k, q}(t)$ - A negative ion with a double-occupied s-orbital $(a=0)$ and two holes in the metal at momenta $k$ and $q$.

The restriction to this trial basis is achieved by projecting the Schrodinger equation $i \frac{d}{d t} \Psi=H \Psi$ onto each sector of the Hilbert space and we obtain the following equations of motion:

$$
\begin{aligned}
i \frac{d}{d t} f & =\sum_{a ; k<k_{f}} V_{a ; k}^{(1) *} b_{a ; k} . \\
i \frac{d}{d t} b_{a ; k} & =\left(\epsilon_{a}^{(1)}-\epsilon_{k}\right) b_{a ; k}+V_{a ; k}^{(1)} f+\delta_{a, 0}(1-1 / N)^{-1 / 2} \sum_{q<k_{f}} V_{0 ; q}^{(2) *}\left[\theta(k-q) d_{k, q}+\theta(q-k) d_{q, k}\right] \\
& +N^{-1 / 2} \sum_{l>k_{f}} V_{a ; l}^{(1)} e_{l k} . \\
i \frac{d}{d t} e_{l, k} & =\left(\epsilon_{l}-\epsilon_{k}\right) e_{l, k}+N^{-1 / 2} \sum_{a} V_{a ; l}^{(1) *} b_{a ; k} . \\
i \frac{d}{d t} d_{k, q} & =\left(2 \epsilon_{0}^{(2)}-\epsilon_{k}-\epsilon_{q}+U\right) d_{k, q}+(1-1 / N)^{-1 / 2}\left(V_{0 ; q}^{(2)} b_{0 ; k}+V_{0 ; k}^{(2)} b_{0 ; q}\right) .
\end{aligned}
$$

The step function $\theta(x)=1$ when $x>0$; otherwise it is zero. Its appearance here is in keeping with the convention of dropping amplitudes $d_{k, q}$ when $k<q$ since they are redundant (ie. $d_{k, q}=d_{q, k}$ ). The logic behind the truncation scheme becomes clear upon considering the nature of the offdiagonal coupling [terms in the Hamiltonian proportional to $N^{-1 / 2}$. These terms couple adjacent sectors of the Hilbert space. (By adjacent we mean sectors that differ by at most one elementary excitation in the band like a hole or a particle-hole pair.) In fact, repeated applications of the atom-metal coupling to the reference state $|0\rangle$ generates all the sectors in the full singlet manybody wavefunction. Now each time $V_{a ; k}$ acts, it brings along a factor of $N^{-1 / 2}$. Thus amplitudes for sectors involving multiple particle-hole pairs are weakly coupled to lower order terms when $\mathrm{N}$ is large. In particular, from Eq. [3.3] it is clear that the amplitude for a single particle-hole pair is reduced by a factor of $N^{-1 / 2}$ in comparison to the amplitudes for the sectors with no particle-hole pairs $\left(f, b_{a ; k}\right.$ and $\left.d_{k, q}\right)$. The probability for a particle-hole pair is therefore reduced by a factor of $1 / N$. By keeping this next-order term one gains insight into the size of the errors produced by the 
truncation of the Hilbert space. It is also possible to estimate the amount of energy lost during the collision process from the formation of particle-hole excitations.

Actually, two other single particle-hole sectors appears at $O\left(N^{-1 / 2}\right)$ in addition to the $|l, q\rangle$ particle-hole sector with its unoccupied atomic orbital. Amplitudes for a particle-hole pair along with singly and doubly occupied atomic orbitals should also be included at this order. Since these new sectors involve amplitudes with respectively three and four momenta indices (the additional indices label the extra holes left behind in the metal when electrons transfer to the atomic orbitals), the numerical task of integrating the equations of motion becomes too taxing (see below) and we drop these sectors from further consideration. In any case, the negative-ion plus particle-hole sector probably contributes little weight because of its high energy. The neglect of the neutral plus particle-hole sector presumably introduces larger errors. Nevertheless, the theory does describe experiments that measure collision energy losses for outgoing positive ions since dissipation occurs via the $|l, q\rangle$ positive ion, particle-hole sector. We take up this analysis in section (IV).

Curiously, upon taking the $N \rightarrow \infty$ limit and eliminating the double-occupied and excited neutral subspaces (by assigning to these sectors very large energies), we find that the equations of motion resemble those of the Heisenberg operators $\hat{c}_{a}(t)$ and $\hat{c}_{k}(t)$ in the Brako-Newns single-particle picture $^{5}$ upon identifying $f \leftrightarrow \hat{c}_{a}$ and $b_{0, k} \leftrightarrow \hat{c}_{k}$. Appearances are deceiving in this case, however, for two reasons. First, equations [3.3] give the time evolution of amplitudes (ie. c-numbers), not operators $^{4}$. The physical meaning of this distinction is as follows: in the $N \rightarrow \infty$ limit of the many-body problem there can be no particle-hole excitations as these amplitudes are suppressed by a factor of $N^{-1 / 2}$. But in the single-particle picture, any number of particle-hole excitations appear because the final state of the system at $t \rightarrow+\infty$ is a Slater determinant built up with creation operators that are themselves linear combinations of the creation operators at the initial time: $|\Psi(\infty)\rangle=\hat{c}_{a}^{\dagger}(\infty) \prod_{k} \hat{c}_{k}^{\dagger}(\infty)|0\rangle$ where $\hat{c}_{a}(t)=\hat{U}(t) \hat{c}_{a}(0) \hat{U}^{\dagger}(t), \hat{c}_{k}(t)=\hat{U}(t) \hat{c}_{k}(0) \hat{U}^{\dagger}(t)$, and:

$$
\hat{U}(t) \equiv \hat{T} \exp \left\{i \int_{0}^{t} d \tau \hat{H}(\tau)\right\}
$$

is the time-evolution operator. 
The second difference between the single-particle picture and the $N \rightarrow \infty$ limit of the manybody equations concerns the sum over momentum in the first of Equations [3.3]: the sum extends only over the momentum $(k)$ of states below the Fermi energy whereas in the single-particle picture the operator that destroys a filled atomic orbital $\left(\hat{c}_{a}\right)$ couples to states both above and below the Fermi surface. One effect of the restriction on $k$ becomes clear upon comparing the final outcomes from different initial conditions to test whether "loss-of-memory" occurs. The "loss-of-memory hypothesis" states that the final state of the outgoing atom should be independent of its initial state if the atom stays in the region of strong coupling to the metal for enough time to erase any memory of the initial state. However, in the $N \rightarrow \infty$ limit, loss-of-memory no longer occurs if the initial incoming state is that of a neutral atom: the electron on the atom cannot jump into a metal state above the Fermi surface (since it is not coupled to those states) but can only fill the single unoccupied state below the Fermi surface (which has vanishing measure in the continuum limit of an infinite number of metal states.) Thus, the atom emerges from the collision in a purely neutral state. In contrast, an incoming positive ion can neutralize because all the electrons below the Fermi surface are available for charge transfer. Upon turning on the coupling to the particle-hole pairs (by returning to the physical case of $N \rightarrow 2$ ), the incoming neutral atoms can now ionize because the valence electron is allowed to transfer into the unfilled levels above the Fermi surface.

This behavior illustrates the importance of electron-hole pairs to the loss-of-memory process. Since we truncate the expansion at the single particle-hole level, perfect loss-of-memory does not occur: the final state occupancies depend to some extent on the initial state. ${ }^{10}$ If more pairs could be included, the loss-of-memory would presumably improve. In practice, we find the discrepancy often to be small; on the other hand one may choose a better initial condition that incorporates the physics of loss-of-memory by starting the integration with the atom-metal system in its ground state at the point of closest approach to the metal (see below). The initial condition is justified both by experiments which show that loss-of-memory occurs and by the single-particle picture where memory of the initial state rapidly dwindles as time progresses along a given trajectory. 
Of course, the equilibrium ground state is an inappropriate starting point if one wishes to study the amount of energy dissipated during the collision process due to the formation of electron-hole pairs which arise during both the inbound and outbound portions of the trajectory. Nevertheless, integrations that start from the equilibrium ground state appear satisfactory for the purposes of making detailed comparisons to experiments that measure the final occupancy probabilities.

The equations of motion are solved numerically by using a finite number, L, of discrete momenta (typically $\mathrm{L}=100$ which means 100 states above and 100 states below the Fermi surface). Because amplitudes $e_{l, k}$ and $d_{q, k}$ have two momenta indices, on the order of tens of thousands of coupled differential equations must be integrated forward along the trajectory. We employ a fourth-order Runge-Kutta algorithm with adaptive time steps. The numerical task is simplified by making a change of variables to remove the diagonal terms from the equations of motion. Let:

$$
\begin{aligned}
f(t) & =F(t) . \\
b_{a ; k}(t) & =B_{a ; k}(t) \exp \left\{i\left[\epsilon_{k} t-\phi_{a}(t)\right]\right\} . \\
e_{l, k}(t) & =E_{l, k}(t) \exp \left\{i\left(\epsilon_{k}-\epsilon_{l}\right) t\right\} . \\
d_{q, k}(t) & =D_{q, k}(t) \exp \left\{i\left[\left(\epsilon_{k}+\epsilon_{q}-U\right) t-2 \phi_{0}(t)\right]\right\} .
\end{aligned}
$$

Here, $\phi_{a}(t) \equiv \int_{0}^{t} \epsilon_{a}(\tau) d \tau$ is the time-evolution phase for the decoupled, but image shifted, atomic orbital. (Recall that the time dependence of $\epsilon_{a}$ comes indirectly from the time-dependent position $z(t)$. For the simple trajectories of Eq. [2.2] and the image shift of Eq. [2.4], the phase integral 
$\phi_{a}(t)$ may be evaluated analytically.) In the new basis we find:

$$
\begin{aligned}
i \frac{d}{d t} F & =\sum_{a ; k<k_{f}} V_{a ; k}^{(1) *} \exp \left\{i\left[\epsilon_{k} t-\phi_{a}(t)\right]\right\} B_{a ; k} \\
i \frac{d}{d t} B_{a ; k} & =V_{a ; k}^{(1)} \exp \left\{i\left[\phi_{a}(t)-\epsilon_{k} t\right]\right\} F \\
& +\delta_{a, 0}(1-1 / N)^{-1 / 2} \sum_{q<k_{f}} V_{0 ; q}^{(2) *} \exp \left\{i\left[\left(\epsilon_{q}-U\right) t-\phi_{0}(t)\right]\right\}\left[\theta(k-q) D_{k, q}+\theta(q-k) D_{q, k}\right] \\
& +N^{-1 / 2} \sum_{l>k_{f}} V_{a ; l}^{(1)} \exp \left\{i\left[\phi_{a}(t)-\epsilon_{l} t\right]\right\} E_{l k} \cdot \\
i \frac{d}{d t} E_{l, k} & =N^{-1 / 2} \sum_{a} V_{a ; l}^{(1) *} \exp \left\{i\left[\epsilon_{l} t-\phi_{a}(t)\right]\right\} B_{a ; k} . \\
i \frac{d}{d t} D_{k, q} & =(1-1 / N)^{-1 / 2} V_{0 ; q}^{(2)} \exp \left\{i\left[\phi_{0}(t)+\left(U-\epsilon_{q}\right) t\right]\right\} B_{0 ; k} \\
& +(1-1 / N)^{-1 / 2} V_{0 ; k}^{(2)} \exp \left\{i\left[\phi_{0}(t)+\left(U-\epsilon_{k}\right) t\right]\right\} B_{0 ; q} .
\end{aligned}
$$

[Actually, for discrete momenta, the amplitude $D_{k, k}$ is a special case that must be treated separately. Factors of $\sqrt{2}$ appear to keep the basis given by Eq. [3.2] normal when $k=q$. For simplicity, we suppress these complicating factors here (which can be neglected in the continuum limit of an infinite number of momenta).] Because the right hand sides of Eqs. [3.6] vanish as the atom and the metal decouple, the equations of motion may be integrated forward in time rapidly when the atom is far from the surface. Probability must be conserved and we check that

$$
1=|F(t)|^{2}+\sum_{a ; k<k_{f}}\left|B_{a ; k}(t)\right|^{2}+\sum_{l>k_{f}, q<k_{f}}\left|E_{l, q}(t)\right|^{2}+\sum_{q<k<k_{f}}\left|D_{k, q}(t)\right|^{2}
$$

remains satisfied to within desired numerical accuracy (typically better than 1 part in $10^{5}$ ) over the entire course of the integration.

We choose either of two initial conditions: (1) start the trajectory far away from the metal surface (see Eq. [2.2]) or (2) from the point of closest approach. In case (1) the initial conditions are given by setting all the initial amplitudes equal to zero with exceptions $F(t=0)=1$ if the incoming atom is a positive ion or $B_{0,0}=1$ if it is neutral. This starting point is used in section (IV d) below to evaluate the energy dissipation due to the formation of particle-hole pairs during the collision. In case (2) the equilibrium ground state of the system is the starting point. (The 
ground state is quickly obtained via the imaginary-time Lanczos algorithm.) This initial condition (which incorporates the physics of complete loss-of-memory) appears to be best for comparisons with experiments that make quantitative measurements of charge fractions ${ }^{10}$ (see below). The double-precision computations are performed on IBM RS/6000 series machines ${ }^{23}$. Depending on the ion velocity, runs take from less than one minute to over an hour of CPU time. Errors introduced by approximating the band continuum with a finite number of states are controlled in the usual manner: (1) runs with twice as many states must yield the same final occupancies to within some tolerance and (2) the final amplitudes $E_{l, k}$ and $B_{a ; k}$ should be smooth functions of the momenta indices. In particular, there must be enough states near the Fermi surface to adequately sample the various amplitudes. We find that 100 states both above and below the band are generally more than sufficient to sample the amplitudes.

The time-evolution of the occupancies in the different many-body sectors for some typical runs are presented in Figure [2]. (In this case, a lithium atom interacts with a $r_{s}=2.6$ jellium surface which has a work function of $4.0 \mathrm{eV}$. The couplings between the atomic and metal states are given below in section IV.) The smoothness of the curves is one sign that enough states have been included in the discrete metal band to adequately emulate the continuum. In Figure $[2(\mathrm{a})]$ the incoming $\mathrm{Li}^{+}$ ion heads inwards towards the metal surface from a starting position $20 \AA$ from the surface and begins to neutralize at around $6 \AA$ when the coupling to the metal becomes sufficiently strong to permit an electron to transfer over to the ion. At approximately $2.8 \AA$ the image shift is sufficiently large to push the $\operatorname{Li}(2 \mathrm{~s})$ level above the Fermi energy. At this point, electron probability begins to transfer back to the metal, increasing the positive ion occupancy. Particle-hole pair formation also becomes appreciable because the electron on the atom can also transfer to a metal level above the Fermi energy. Only at the closest distances $(z<2 \AA)$ does the negative ion occupancy become appreciable. At these distances the affinity level drops below the Fermi level. Apparently the negative sector competes with the neutral and positive sectors at short distances, because the occupancy in the neutral and positive sectors drops close in. The excited neutral $2 \mathrm{p}_{z}$ channel (not 
shown) also becomes active at short distances. On the outward leg of the journey, the positive channel continues to grow until it reaches $2.8 \AA$ again and then electron probability once again dumps back onto the atom, increasing the neutralization probability. Finally, around $6 \AA$ the occupancies settle down. We call this distance the "settling distance" for the $\operatorname{Li}(2 \mathrm{~s})$ orbital. This distance is to be distinguished from the "freezing distance" which has been defined ${ }^{24}$ to be the distance where the charge state is determined.

In Figure [2a] the final probability for a particle-hole pair to be formed during the interaction is about $4 \%$. An average of $0.036 \mathrm{eV}$ is dissipated due to these pairs. Negative ion and excited neutral production at the relatively large work function of $4.0 \mathrm{eV}$ is negligible; these channels empty quickly as the atom leaves the region of strong coupling. As expected, particle-hole production is suppressed in Figure [2(b)] (the particle-hole probability is about $0.2 \%$ and the average dissipated energy is only $0.002 \mathrm{eV}$ ) since in this case the system starts from the equilibrium ground state at the point of closest approach. This low energy initial state is not conducive to the formation of energetic particle-hole pairs. It seems possible that the smaller occupancy in the particle-hole sector for this initial condition increases the accuracy of our particle-hole expansion and thus justifies our use of this initial condition for comparisons with the charge state experiments. Note that the two different initial conditions yield similar final occupancies, to within about $13 \%$, for the positive and neutral fractions, demonstrating that significant loss-of-memory occurs despite the truncation of the Hilbert space at the one particle-hole level.

The occupancy in the particle-hole channel for particle-hole pairs of different energies peaks near $0.6 \mathrm{eV}$ for the run displayed in Figure [2(a)]. A peak in the particle-hole energy distribution is a generic feature of our many-body theory; different parameters, however, change the value of the peak energy. Similar peaks (and energy dissipations) were found in the single-particle approximation of $\operatorname{Ref}[25]$ and the calculation of $\operatorname{Ref}[12]$. 


\section{PRELIMINARY COMPARISON WITH EXPERIMENT}

In order to make contact with the experiments described below, we make use of first-principles calculations of the couplings between the atomic and metal states. We first assume that copper is adequately described by $r_{s}=2.6$ jellium metal. We then use level widths $\Delta_{a}(z)$ for neutral alkali atoms calculated as in $\operatorname{Ref}[13]$. (Actually, the values reported in $\operatorname{Ref}[13]$ are for $r_{s}=2.0$. The widths calculated for $r_{s}=2.6$ are very similar and these are the ones we use $^{26}$.) For the negative ion width we use values recently calculated for $r_{s}=2.0$ by Nordlander ${ }^{14}$. Similar values are also found via the coupled angular mode $(\mathrm{CAM}) \operatorname{method}^{27}$. Next, we relate these level widths to the couplings $V_{a}(z)$ by the usual single-particle Fermi Golden-Rule formula: $\pi N L V_{a}^{2}=\Delta_{a} D$ where $\mathrm{D}=4.0 \mathrm{eV}$ is approximately the half-bandwidth of copper. (This formula already incorporates the

factor of $N^{\frac{1}{2}}$ that appears in the Hamiltonian [2.1].) By setting $\mathrm{L}=100$ and fitting $V_{a}(z)$ to the form of Eq. [2.7] we obtain the following parameters (all in atomic units).

(1) Lithium: coupling to the $\operatorname{Li}(2 \mathrm{~s})$ state.

$$
\begin{array}{r}
\tilde{V}=\exp (-2.399), \\
a_{-1}=-3.881, \\
a_{1}=-0.4916 .
\end{array}
$$

Coupling to the $\operatorname{Li}\left(2 \mathrm{p}_{z}\right)$ state.

$$
\begin{gathered}
\tilde{V}=\exp (-4.183), \\
a_{-1}=-0.7205, \\
a_{1}=-0.2346 .
\end{gathered}
$$

Coupling to the $\mathrm{Li}^{-}\left(2 \mathrm{~s}^{2}\right)$ state.

$$
\begin{array}{r}
\tilde{V}=\exp (-5.084), \\
a_{-1}=+1.529, \\
a_{1}=-0.1669 .
\end{array}
$$


(2) Sodium: coupling to the $\mathrm{Na}(3 \mathrm{~s})$ state.

$$
\begin{array}{r}
\tilde{V}=\exp (-2.121), \\
a_{-1}=-5.557, \\
a_{1}=-0.4877 .
\end{array}
$$

Coupling to the $\mathrm{Na}\left(3 \mathrm{p}_{z}\right)$ state.

$$
\begin{array}{r}
\tilde{V}=\exp (-3.062), \\
a_{-1}=0.531, \\
a_{1}=-0.1773 .
\end{array}
$$

Coupling to the $\mathrm{Na}^{-}\left(3 \mathrm{~s}^{2}\right)$ state.

$$
\begin{array}{r}
\tilde{V}=\exp (-5.151), \\
a_{-1}=1.677, \\
a_{1}=-0.1559 .
\end{array}
$$

The geometry and symmetry of the $p_{x}$ and $p_{y}$ orbitals suggests that their coupling to the metal is small; this is borne out by the jellium calculations ${ }^{13}$. We ignore them in our analysis.

In the following four subsections we explore some consequences of our many-body theory, keeping in mind the possibility that different parameters could provide a better description of the observations. (Nevertheless, these couplings serve as a standard of comparison for future studies.) We start our discussion with a quantitative test of the theory: neutralization from a clean surface. Local inhomogeneities in the surface potential are small for a clean surface, making comparison with theory relatively easy. We then consider two experiments that directly test the many-body features of our theory: the detection of excited neutrals and negative ions. Finally, we discuss the formation of particle-hole pairs. In this case even qualitative comparisons are difficult; we can only show that the predictions of the theory are consistent with the experimental upper bound on energy dissipation. 


\section{A. Neutralization by a Clean Copper Surface}

We measure neutralization probabilities for $\mathrm{Li}, \mathrm{Na}$ and $\mathrm{K}$ scattered from clean $\mathrm{Cu}(001)$ along the $\langle 100\rangle$ azimuth for a range of scattered atom velocities. Clean surfaces offer the advantage of minimizing electrostatic inhomogeneities that complicate the interpretation of results. Energetic considerations show that the adiabatic charge states for $\mathrm{Li}$ and $\mathrm{Na}$ in the $\mathrm{Li}+\mathrm{Cu}$ and $\mathrm{Na}+\mathrm{Cu}$ systems are neutral when the atoms are far from the surface, while for the $\mathrm{K}+\mathrm{Cu}$ system the $\mathrm{K}$ is positively ionized. We find that the $\mathrm{Li}$ and $\mathrm{K}$ monotonically approach the adiabatic charge states as the scattered atom velocity decreases. However, for $\mathrm{Na}$ the neutralization probability is nonmonotonic; it initially decreases with decreasing velocity and then increases, approaching the adiabatic ground state only at the lowest velocities.

The experiments were performed in an ultra high vacuum (UHV) system. The experimental techniques are described elsewhere. ${ }^{28,29,30}$ Only the relevant details are presented here. In the experiments described in this section, we used $\mathrm{Li}, \mathrm{Na}$, and $\mathrm{K}$ ions with incident energies from $5 \mathrm{eV}$ to $1600 \mathrm{eV}$. All measurements were performed on a clean $\mathrm{Cu}(001)$ surface, prepared by standard sputter and anneal cycles. The surface order and cleanliness were checked with low energy electron diffraction (LEED) and Auger electron spectroscopy (AES), respectively. The scattered atoms are detected with a time-of-flight (TOF) spectrometer, mounted on a rotatable platform, which can be used to make velocity- and angle-resolved measurements of neutral and positively and negatively ionized alkalis. ${ }^{30}$ This detector can be operated in a mode whereby the velocity- and angle-resolved neutralization probabilities for the scattered alkalis can be determined.

In Figure [3] we show the measured neutralization probability of lithium and sodium scattering off of a clean $\mathrm{Cu}(001)\langle 100\rangle$ surface as a function of perpendicular component of the outgoing atomic velocity. The neutralization as a function of perpendicular velocity $P^{0}(v)$ is qualitatively different for each species. For Li, the neutralization monotonically decreases as the velocity increases and $0.25 \leq P^{0} \leq 0.75$ for the velocities investigated. (For $\mathrm{K}$, essentially no neutralization is found.) For $\mathrm{Na}$, the neutralization versus perpendicular velocity has a minimum and $0.04 \leq P^{0} \leq 0.15$ in 
the velocity range investigated.

Figure [3] also shows predictions for the neutralization probability of lithium and sodium from both the many-body model and the standard single-particle model ${ }^{3}$ (with the same couplings as the many-body model, but now of course only between the neutral s-orbital and the metal). Both theories reproduce the experimental trends. The origin of these trends becomes clear if we consider the different lifetimes and energies of the $\mathrm{Li}(2 \mathrm{~s}), \mathrm{Na}(3 \mathrm{~s})$, and $\mathrm{K}(4 \mathrm{~s})$ states. For potassium, the first ionization energy is $4.34 \mathrm{eV}$. Since the image potential increases the energy of the $\mathrm{K}(4 \mathrm{~s})$ level, it lies predominantly above the Fermi level, and it is energetically favorable for the $\mathrm{K}(4 \mathrm{~s})$ level to be empty over a wide range of atom-surface separations. Thus, almost no neutralization of $\mathrm{K}^{+}$will occur. Indeed, the many-body theory also predicts little neutralization. Lithium, on the other hand, has an ionization energy of $5.39 \mathrm{eV}$ and the neutralization probability decreases as the velocity increases. The reason for this decrease is clear: The $\operatorname{Li}(2 \mathrm{~s})$ level lies below the Fermi energy when the atom is far from the surface; only close to the surface is it image shifted above the Fermi energy. In the velocity range of the experiment, the freezing distance decreases as the outgoing velocity increases, enhancing the positive fraction.

Sodium is intermediate between these two cases. The ionization energy is $5.14 \mathrm{eV}$, so the $\mathrm{Na}(3 \mathrm{~s})$ resonance is, as in the case of lithium, predominantly below the Fermi level far from the surface and predominantly above it close to the surface. However, because the $\mathrm{Na}(3 \mathrm{~s})$ resonance is closer to the Fermi level there is considerably less neutralization than for lithium and the neutralization does not monotonically decrease as the scattered velocity increases. The minimum observed in Figure [3] is due to the approximately exponential increase in the level width with decreasing atom-surface separation. For sodium, this increase is relatively more important at higher velocities than the shift in the energy due to the image potential. Thus, as the velocity increases and the freezing distance decreases, even though the resonance is at a higher energy, more of the resonance lies below the Fermi level and the neutralization increases.

The curves for the many-body and single-particle models shown in Figure [3] are qualitatively 
similar. However, for both $\mathrm{Li}$ and $\mathrm{Na}$, the many-body model predicts less neutralization at all velocities. Four possible reasons for the differences are given here. First, differences between the two models indicate that inter-atomic correlations are important, even in the dynamics of alkali scattering from clean metal surfaces. Consider the case of Li scattering from clean $\mathrm{Cu}(001)$. Different Li resonances, for example $\operatorname{Li}(2 \mathrm{~s})$ and $\operatorname{Li}(2 \mathrm{p})$, have different couplings to the surface and result in different freezing distances along the outgoing trajectory. The freezing distance for the lowest energy state (the ground state neutral) is typically the smallest. Thus, even at the distance where the occupation of the $\operatorname{Li}(2 \mathrm{~s})$ state is frozen substantial occupation of the excited neutral state, $\operatorname{Li}(2 p)$, can remain. In fact, the many-body theory predicts that the occupation of the $\operatorname{Li}(2 \mathrm{p})$ state can be as high as $\sim 4 \%$ at the settling distance of the $\operatorname{Li}(2 \mathrm{~s})$ state. As the atom moves further away from the surface, charge transfer from the $\operatorname{Li}(2 \mathrm{p})$ can now occur leaving the atom in a positively ionized state. (Virtually no excited neutrals or negative ions survive far from the surface.) Thus, if we ignore the role of spin, excited states and other channels (single-particle picture) we will obtain incorrect occupation probabilities.

Second, the level widths used in the many-body model were calculated in a single particle picture. ${ }^{13,14}$ The use of these widths in our many-body theory is not necessarily justified. Indeed, if we calculate the lifetimes of the various atomic states in our many-body theory (by holding the atom at a fixed distance $z$ from the surface), we obtain different widths than those obtained via Fermi's Golden Rule which of course ignores correlations. To see this, we appeal to similarities in the $N \rightarrow \infty$ limit between our many-body equations and the equations for the time-evolution of the operators in the single-particle picture mentioned above in section (III). The equations are similar only for atomic levels deep below the Fermi energy. Atomic widths for these levels calculated in either picture are the same in the $N \rightarrow \infty$ limit. But upon taking the physical $N \rightarrow 2$ limit we find additional terms (the particle-hole amplitudes) arise in the many-body picture. Thus atomic lifetimes calculated in the two pictures will generally differ. Perhaps a more sensible approach would be to renormalize the metal-atom couplings $V_{a ; k}$ to make the many-body theory reproduce 
the lifetimes calculated within the single-particle picture. Investigations along these lines may shed some light on the effect of many-body correlations on atomic lifetimes.

Third, the parallel velocity of the scattered atom (which shifts the Fermi surface in the atomic reference frame and changes the couplings) must be incorporated into the model to obtain good quantitative agreement between theory and data ${ }^{30}$. The parallel velocity effect is significant even at surprisingly small velocities $\left(v \approx 0.01 v_{f}\right)$ and non-glancing scattering geometries (e.g. $\left.\theta_{f}=45^{\circ}\right)$.

Finally, our solution of the many-body model is approximate. As mentioned above, the final occupancies depend to some extent on the initial conditions. This dependence on the initial conditions represents a limitation of the approximate solution of the model since good experimental and theoretical evidence exists for complete loss-of-memory.

Our comparison of the single-particle and many-body models demonstrates that in the case of $\mathrm{Li}, \mathrm{Na}$ and $\mathrm{K}$ scattered from $\mathrm{Cu}(001)$ both theories agree qualitatively with experiment. Further comparison of experimental results with theoretical predictions utilizing somewhat different parameters should provide additional quantitative insight into the strengths of the couplings between the metal and the atomic states. Other experiments at lower work functions highlight the differences between the single-particle and many-body pictures. We describe two such experiments below. 


\section{B. Excited States}

We performed a number of experiments which directly test the many-body aspects of our theory of resonant charge transfer. Using the TOF neutral spectrometer mentioned above and low level photon counting techniques, we measured the work function dependence of both the relative yields of excited states and the charge state fractions resulting from the scattering of low and hyperthermal energy beams of alkali ions off an alkali-covered $\mathrm{Cu}(001)$ surface. Production of these species is enhanced at low work functions and high velocities (which shorten the freezing distances and thereby increase the final occupancies of energetic states). The theory predicts all of the qualitative trends exhibited by the experimental data.

One feature of the theory presented here is that it predicts the probability with which incident ions are scattered into neutral excited states. In this section, we compare our theoretical predictions to measurements of the relative $\mathrm{Li}(2 \mathrm{p})$ yield produced when $\mathrm{Li}^{+}$strikes a $\mathrm{Cu}(001)$ surface with sub-monolayer coverage of potassium adsorbates, hereafter denoted as a $\mathrm{K} / \mathrm{Cu}(001)$ surface. We measure the dependence of this yield on the work function shift that is induced by depositing $\mathrm{K}$ onto the $\mathrm{Cu}(001)$ surface. ${ }^{31}$ Measurements of this type have been made previously for $\mathrm{Li}^{+}$incident on $\mathrm{Cs} / \mathrm{W}(110) \cdot{ }^{32}$

To measure the relative yields of excited states, we collect the photons which are emitted during the decay of these states. The photons are transported by a fiber optic cable and counted by using a photomultiplier. Line filters corresponding to particular optical transitions can be inserted into the light path to isolate the various excited states.

For the measurements presented here, the energy of the impinging $\mathrm{Li}^{+}$ions is $400 \mathrm{eV}$ and the incident angle (measured with respect to the surface normal) is $65^{\circ}$. The incident beam is directed along the $\langle 100\rangle$ azimuth. Ions which are scattered into the $\operatorname{Li}(2 \mathrm{p})$ state survive in the $\operatorname{Li}(2 \mathrm{p})$ state for a half-life of $27 \mathrm{nsec}$ and decay to the $\operatorname{Li}(2 \mathrm{~s})$ state by emitting a photon $(E=1.85 \mathrm{eV}$, and $\lambda=673 \mathrm{~nm})$. Thus, the ions scattered into the $\operatorname{Li}(2 \mathrm{p})$ state are detected by collecting the photons corresponding to the $\operatorname{Li}(2 \mathrm{p}) \rightarrow \operatorname{Li}(2 \mathrm{~s})$ transition. 
The single-particle picture of charge transfer, ${ }^{3}$ shown schematically in Figure [4] for the closed $\mathrm{Li}$ atom and clean $\mathrm{Cu}(001)$ system with work function $\Phi=4.59 \mathrm{eV}$ (this absolute value was determined in $\operatorname{Ref}[33])$ shows that little scattering into the $\operatorname{Li}(2 \mathrm{p})$ state and other, higher-lying excited states is expected because these states are not resonant with any occupied states in the metal. However, as the work function decreases, the occupied metallic states are brought into resonance with the excited states. Decreasing the work function therefore increases the yield of excited atoms scattered from the surface. Since the energy of the $\operatorname{Li}(2 \mathrm{~s})$ state is significantly lower than that of the $\operatorname{Li}(2 \mathrm{p})$ state, we expect the fraction of atoms scattered into the $\operatorname{Li}(2 \mathrm{~s})$ state to be much larger than the fraction of atoms scattered into the $\operatorname{Li}(2 \mathrm{p})$ state. In principle, excited states of higher energy may also participate but should not constitute a significant fraction of the excited states in the scattered flux. (We have verified that greater than $90 \%$ of the measured emitted light in the optical range is due to the $\operatorname{Li}(2 \mathrm{p}) \rightarrow \operatorname{Li}(2 \mathrm{~s})$ transition.) However, the affinity level, also shown in Figure [4], will also be increasingly populated as the work function decreases. Competition between the $\operatorname{Li}(2 \mathrm{p})$ and $\mathrm{Li}^{-}\left(2 \mathrm{~s}^{2}\right)$ (i.e., negative ion) channels therefore should occur at low work functions.

In Figure [5], we plot the relative yield of $\mathrm{Li}^{+}$ions which are scattered into the $\mathrm{Li}(2 \mathrm{p})$ state versus the work function shift induced by the deposition of K. In the same figure, we plot the predicted yield of $\operatorname{Li}(2 \mathrm{p})$ at the maximum outgoing normal velocity since (as explained above) these trajectories are responsible for most of the excited states. The theoretical results are normalized to the experimental results (we comment on the absolute numbers below). Note that the peak values of the measured and predicted yields occur at nearly the same value of the work function shift, $\Delta \Phi \approx-1.8 \mathrm{eV}$, and that the peak in the measured yield is broader than that predicted by the theory.

The peak in the predicted yield is due to competition between the $\mathrm{Li}(2 \mathrm{p})$ state and the $\mathrm{Li}^{-}\left(2 \mathrm{~s}^{2}\right)$ state. It seems that a balance between these minority state populations obtains at work function values near $2.6 \mathrm{eV}$. This balance determines the work function value at which the peak in the 
predicted $\operatorname{Li}(2 \mathrm{p})$ yield occurs. Our work indicates that this value is relatively insensitive to the parameters we use in the many-body theory. Other experiments on similar systems suggest that Auger processes may also play a role. ${ }^{32}$ We note that both mechanisms could be operating: Auger neutralization may be occuring along the incoming portion of the trajectory, but memory of the neutralization history will be erased as the atom enters the strong coupling region. On the outgoing trajectory, the different resonant processes described by our theory are consistent with experiments we have performed to date. Future extensions of the many-body theory that include Auger amplitudes will address the question of the relative importance of Auger versus resonant processes.

One likely explanation for the discrepancy between the widths of the experimentally observed and theoretically predicted peaks is our failure to account for local variations in the electrostatic potential induced by the alkali adsorbates in the model. Such variations tend to smear out workfunction dependence of observable quantities like the neutralization probability ${ }^{17,3}$. Also, the couplings between the atomic states and the metal electrons may be altered significantly in the vicinity of an adsorbate atom. Local variations can be incorporated into the model by averaging results over many possible ion trajectories that impact the surface at different points and thus sample different electrostatic potentials and couplings.

Finally, we estimate the fraction of atoms scattered into the $\operatorname{Li}(2 \mathrm{p})$ state to be of order 0.004 at $\Delta \Phi \approx-1.8 \mathrm{eV}$. The peak occupancy of the $2 \mathrm{p}_{z}$ state predicted by the model is 0.026 . The predicted peak value is quite sensitive to the atomic state lifetimes and energies near the surface. Different (but still reasonable) values for these parameters change the excited state fraction by an order of magnitude. 
To measure the charge state fractions in the flux scattered into a particular final angle, we use the TOF spectrometer. Recall that it permits discrimination and detection of alkali particles with different charge states. We measure the work function dependence of charge state fractions in the scattered flux when $\mathrm{Li}^{+}$ions impinge on $\mathrm{Cs} / \mathrm{Cu}(001) .{ }^{31}$ In our experiments, we direct a 400 $\mathrm{eV} \mathrm{Li}^{+}$ion beam toward the $\mathrm{Cs} / \mathrm{Cu}(001)$ surface and along the $\langle 100\rangle$ azimuth, with an incident angle of $65^{\circ}$. The final angle of the scattered particles was $64^{\circ}$. The work function shift induced by the deposition of Cs on the surface is measured with a Kelvin probe. Similar measurements, but without velocity resolution, were reported previously. ${ }^{17}$

Figure [6] is a plot of the measured charge state fractions versus the work function shift induced by Cs adsorption. When the surface is clean, the only charge states in the scattered flux are the positive ion state and the neutral states; no negative ions are present to within the experimental uncertainty of a few percent. As the work function shift decreases from zero, (in other words, as the work function decreases), the positive ion fraction decreases; a corresponding increase is seen in the neutral fraction. For work function values greater than approximately $2.6 \mathrm{eV}$, the negative ion fraction is less than a few percent. The negative ion fraction becomes appreciable only for work function values less than $2.6 \mathrm{eV}$, with a maximum value of 0.14 . In the range of work function values for which the negative ion fraction is appreciable, the positive ion fraction does not exceed a few percent.

Figure [6] shows that the qualitative trends displayed by the charge state fractions are reproduced by the model. We can qualitatively understand these trends within the one-electron picture. First, consider the work function dependence of the positive ion fraction. As the work function decreases, more and more of the atomic resonance corresponding to the $\operatorname{Li}(2 \mathrm{~s})$ level lies below the Fermi level, leading to its increased population (smaller positive ion fraction). We can also construct a one-electron picture for the affinity level ${ }^{34}$. As the work function decreases, an increasing portion of the atomic resonance corresponding to the $\mathrm{Li}^{-}\left(2 \mathrm{~s}^{2}\right)$ level lies below the Fermi level, and 
it should be increasingly populated and more negative ions emerge from the collision. Note that the slope of the measured ion fraction versus the work function is smaller than that predicted by the model. As in the previous section this is consistent with the neglect of local variations in the electrostatic potential induced by the adsorbates ${ }^{3,17}$.

The above results are similar to those obtained by Geerlings et al. for $\mathrm{Li}^{+}$scattering from $\mathrm{Cs} / \mathrm{W}(110)$. In experiments by Brenten et al. for $\mathrm{Li}^{+}$incident on $\mathrm{Cs} / \mathrm{W}(110)$, the relative yields of $\mathrm{Li}^{+}$and $\mathrm{Li}^{-}$have been measured as a function of Cs coverage, along with the yields of $\mathrm{Li}(2 \mathrm{p})$ and emitted electrons. ${ }^{35}$ In addition, Ashwin and Woodruff have reported measurements of the ratio of positive ion fractions for $\mathrm{Li}^{+}$scattering from $\mathrm{Cu}$ and $\mathrm{Cs}$ when $\mathrm{Li}^{+}$is incident on $\mathrm{Cs} / \mathrm{Cu}(110) .{ }^{36}$

In summary, many final atomic states occur when $\mathrm{Li}^{+}$scatters from a Cs-covered copper surfaces. We observe $\mathrm{Li}^{+}, \mathrm{Li}(2 \mathrm{~s}), \mathrm{Li}(2 \mathrm{p})$, and $\mathrm{Li}^{-}\left(2 \mathrm{~s}^{2}\right)$. Auger processes also play a role in charge transfer in these systems. ${ }^{37}$ We discuss this channel and its incorporation into the many-body model (something not possible in single-particle models) in the conclusion. 


\section{Dissipation Due to the Formation of Particle Hole Pairs}

The importance of particle-hole pair formation in the scattering of alkali ions from metal surfaces can be estimated by comparing the measured final energies of ions scattered from a metal surface at incident energies ranging from a few $\mathrm{eV}$ to a few $\mathrm{keV}$ to the final energies predicted by classical trajectory simulations. We assume here that resonant charge transfer is the only significant mechanism for the production of particle-hole pairs. If, as the $1 / \mathrm{N}$ particle-hole expansion assumes, particle-hole pair production is limited then trajectory simulations which do not include energy loss to particle-hole pair production should be able to reproduce the measured energy loss in ion-surface collisions. In this section we describe experiments (for more details see Ref[16]) which make this comparison and which lend credence to the assumption that particle-hole pair production is limited.

We measured a series of energy spectra for $\mathrm{Na}^{+}$scattering from $\mathrm{Cu}(001)$ along the $\langle 100\rangle$ azimuth at an incident angle of 45 degrees and a final angle of 45 degrees as measured from the surface normal. The scattering takes place within the plane defined by the $\langle 100\rangle$ azimuth and the surface normal. Trajectory simulations indicate that these energy spectra contain contributions from four different kinds of scattering trajectories. The highest energy peak contains contributions from two different types of trajectories that coincidentally scatter with nearly the same final energy, namely the quasi-double (QD) and triple zig-zag (TZZ) trajectories. The middle and lowest energy peaks consist of double zig-zag (DZZ) and quasi-single (QS) trajectories, respectively. The QS and QD trajectories involve momentum transfer to atoms which lie along a $\langle 100\rangle$ chain. The QS trajectory transfers momentum to primarily one surface atom and the QD trajectory transfers momentum to two adjacent surface atoms in the chain. The zig-zag trajectories scatter from atoms in adjacent $\langle 100\rangle$ chains. The DZZ and TZZ trajectories involve respectively two or three surface atoms.

The relative cross sections (peak heights) and fractional energy losses (peak energies) in the measured energy spectra change as a function of incident energy. Both of these trends are reproduced quantitatively using the classical trajectory simulation code SAFARI ${ }^{38}$. SAFARI integrates Hamilton's equations of motion for the ion interacting with the surface. The ion-surface interaction 
potential is one of the input parameters in the simulation. The energy and angular distributions of 10 to $100 \mathrm{eV} \mathrm{Na}^{+}$scattering from $\mathrm{Cu}(001)\langle 100\rangle$ are reproduced quantitatively using an interaction potential which consists of a sum of two terms. The first contribution is a repulsive potential that is modeled as a sum of Hartree-Fock pair potentials where the sum runs over six or more surface atoms closest to the scattering ion. To this repulsive term a second, attractive, potential is added (see Eq. [2.4]) to account for the image interaction between the ion and surface. The only free parameters in the potential are $V_{\max }$ and the position of the image plane. The values of these parameters $\left(V_{\max }=2.6 \mathrm{eV}\right.$ and the image plane is set at a distance $0.8 \AA$ beyond the first plane of copper nuclei) are determined by requiring that the simulated energy and angular distributions agree with the data.

In Figure [7] the ratio of the scattered energy to the incident energy, $E / E_{0}$, of the peaks in the measured energy spectra are plotted as a function of the incident energy $E_{0}$. Also shown are the values of the corresponding scattered trajectories calculated in the simulations (solid triangles and line). The measured energies are uncertain to within $\pm 0.5 \mathrm{eV}$ due to contact potential differences within the apparatus. The only energy loss mechanism included in these simulations is momentum transfer from the scattering ion to the recoiling surface atoms. It must be noted that we assume that the ion-surface potential is accurately modeled in our simulation ${ }^{16}$, since an increase in the depth of the attractive well could mimic energy loss due to particle-hole formation. With this in mind, the excellent agreement between the measured and simulated energy loss of these trajectories suggests that the additional energy transfer from the ion to the surface due to particle-hole pair excitations in the metal is very small. This is consistent with the theoretical conclusion that the energy dissipated due to the formation of particle-hole pairs is limited to less than a few tenths of an electron volt. Thus we have some direct experimental evidence that the systematic expansion in the number of particle-hole pairs is well behaved. 


\section{CONCLUSION}

The preceding sections describe a generalized Newns-Anderson model and its systematic solution. The theory goes beyond earlier work in that it incorporates electron spin, Coulomb repulsion, level crossings, particle-hole pairs, and excited atomic states all within one systematic framework. Results obtained are highly encouraging. In particular the theory reproduces the trends in the neutralization probabilities of $\mathrm{Li}, \mathrm{Na}$, and $\mathrm{K}$ ions that scatter off clean $\mathrm{Cu}(001)$ surfaces. It also agrees qualitatively with the measured negative ion fractions of $\mathrm{Li}$ and $\mathrm{Na}$ ions that interact with low work function surfaces. For the case of lithium the theory predicts the existence of a peak in the intensity of the optical $2 \mathrm{p} \rightarrow 2 \mathrm{~s}$ transition as a function of the surface work function and this peak has now been seen in our experiments.

A number of fascinating questions can be posed within this framework. These questions can be answered by extending the existing model and its solution to more general situations:

(1) Experiments with other ion species, such as atomic oxygen ${ }^{39}$, call for theoretical attention. The incorporation of additional orbitals and initial states with different orbital angular momentum into the Newns-Anderson model and our systematic solution is straightforward and will not slow down the numerical integrations significantly. A theory of Oxygen using the slave-Boson formalism was presented recently ${ }^{40}$.

(2) The incorporation of more realistic target band structure into the model is also fairly simple. For example, surface states can be included as a separate metallic band. In addition, experiments on semiconducting targets have been done $^{41}$ and these measurements should be reexamined using the many-body theory.

(3) Related to the nature of the band structure are the effects of the parallel component of the ion velocity ${ }^{2,42}$ and the local electronic structure induced by adsorbates ${ }^{3}$. The band structure of the target and the atom-metal matrix elements $V_{a ; k}(z)$ should be recomputed in the boosted reference frame of the ion. This effect has already been studied within the single-particle picture ${ }^{15}$ and it would be worthwhile to include these effects in the many-body model. Local variations in the 
electrostatic potential due to the adsorbates also should be included in the calculation to permit more quantitative comparisons with experiment. Finally, how do many-body effects change the overlap matrix elements $V_{a ; k}$ between the atom and the target metal? Can experiments with clean surfaces further constrain these parameters?

(4) We can include Auger processes in the many-body theory, and now need to think about how to model these amplitudes in a meaningful way. One danger to be avoided is the introduction of more and more parameters into the theory without a clear understanding of their values. There is provocative theoretical ${ }^{43}$ work on Auger processes which we can draw upon to find sensible models for the amplitudes. In addition, experiments have found secondary electrons resulting from ionsurface collisions which are consistent with various Auger processes, ${ }^{44,35,37}$ but the determination of absolute cross sections is a difficult experimental problem. The Auger term complicates the solution of the many-body equations significantly because it involves three momenta indices. Nevertheless, preliminary work indicates that the numerical problem remains tractable as long as L, the number of metal states, is not too large.

Questions outside the framework presented here include:

(5) How does the systematic $1 / \mathrm{N}$ solution compare to the Langreth-Nordlander ${ }^{6}$ slave-Boson approach? As both theories start from the same basic model, the question concerns the limitations of the approximations made in the $1 / \mathrm{N}$ and slave-Boson solutions. A preliminary comparison has shown that qualitatively similar behavior is exhibited by both theories, in that both "lock out" additional charge transfer in the limit of large Coulomb repulsion as described in the introduction $[\operatorname{section}(\mathrm{I})]$.

(6) How do the electron-electron interactions inside the target metal affect charge-transfer processes? Is the Landau Fermi liquid approach adequate? Finite quasi-particle lifetimes may play a significant role in the particle-hole sector of the theory. It should at least be possible to include phenomenological lifetimes in the many-body theory. One class of interesting systems is the heavy Fermion materials. The extremely narrow bandwidths (due to large effective electron 
masses) should enhance the formation of particle-hole pairs and strong collective phenomena may occur. In a more speculative vein, recent work shows that resonant tunneling in one-dimensional Luttinger liquids exhibits anomalous behavior ${ }^{45}$. If, as has been suggested in the case of the hightemperature superconductors, Luttinger liquids are realized in higher dimensional materials, would any clear signatures appear in charge transfer experiments?

(7) What are the limitations of the Newns-Anderson model? In particular, we know that the atomic orbitals are distorted and hybridized as the ion approaches the surface. Do new resonances appear? Should explicit matrix elements be added to model direct hybridization? Analysis of experiments on static adsorbed atoms might yield insight into these questions.

(8) Can the asymptotic formalism ${ }^{46}$ of Dorsey et al. be incorporated into the $1 / \mathrm{N}$ solution? This approach might permit more efficient solutions of the dynamical equations. It involves a calculation, within the $1 / \mathrm{N}$ approximation, of the static eigenstates of the system at variable distance $z$. The dynamical problem can then be solved, for a series of different perpendicular velocities, using these states (which need only be evaluated once). We already calculate the ground state of the system at the point of closest approach; perhaps only low-lying states are needed for an accurate description of the dynamics.

\section{Acknowledgements}

We thank A. Dorsey, D. Goodstein, D. Langreth, P. Nordlander, J. Sethna, and E. Zaremba for helpful discussions. We also thank P. Nordlander for providing us with results prior to publication. This research was supported by an IBM postdoctoral fellowship (J.B.M), the Cornell Materials Science Center and the National Science Foundation (grant nos. NSF-DMR-9022961 and NSFDMR-9121654). D.R.A. was also supported by the Swedish Institute and the Sweden-America Foundation. Additional support for this research was provided by the Air Force Office of Scientific Research (grant no. AFOSR-91-0137). 


\section{FIGURE CAPTIONS}

(1) Schematic of the four sectors kept in the variational many-body wavefunction. The Fermi energy is denoted $\epsilon_{f}$ and the circle is the hyperthermal atom. State $\mid 0>$ represents a positive ion with a closed inner shell and an unperturbed Fermi liquid. State $\mid a ; k>$, on the other hand, is a neutral atom with orbital $a$ filled plus a hole at momentum $k$ in the metal. It is obtained from the state $\mid 0>$ by the transfer of an electron from state $k$ in the metal to the atomic orbital $a$ (arrow). State $\mid l, k>$ is a particle-hole pair: the electron has moved from momentum $k$ (creating a hole) to momentum $l$ via a hop to and from the atom. It is vital to include this next-order term in the calculation because it plays an important role in the loss-of-memory of the initial incoming state (see text). Finally, $\mid k, q>$ is the state of a negative ion - the two valence electrons are in the lowest orbital - with two holes of momenta $k$ and $q$ left behind in the metal.

(2) Occupancy of each sector of the $N=2$ many-body wavefunction as a function of the atomic position. Here a lithium atom interacts with a metal surface of work function $\Phi=4.0 \mathrm{eV}$. The band consists of 100 states above and 100 states below the Fermi surface with a full bandwidth of $8 \mathrm{eV}$ and constant density of states. The couplings are determined from the atomic lifetimes calculated in the single-particle approximation of Ref[13] and Ref[14] (see section IV). (a) A positive ion heads inward towards the metal at $z_{f}=20.0 \AA$ with initial perpendicular velocity $u_{i}=0.04 \mathrm{au}$, bounces at $z_{0}=1.0 \AA$, and then departs at a lower velocity of $u_{f}=0.03 \mathrm{au}$. The final occupancy probabilities are: $P^{+}=0.2150$ (with and without a particle-hole pair), $P^{0}=0.7838$ (with virtually no excited states), $P^{-}=0.0011$ and the probability for one particle-hole to be formed during the collision is 0.0439. (b) Trajectory leaves the equilibrium ground state at the point of closest approach with a velocity of $u_{f}=0.03 \mathrm{au}$. The final occupancy probabilities are: $P^{+}=0.3453$ (with and without a particle-hole pair), $P^{0}=0.6546$ (also with no excited states), $P^{-}=0.0001$ and the probability for one particle-hole to emerge 
from the equilibrium ground state is 0.0024 .

(3) Measured and predicted neutralization probability $P^{0}$ of lithium and sodium that scatter off of a clean $\mathrm{Cu}(001)$ surface. The neutral fraction is plotted as a function of perpendicular velocity. The scattering geometry is depicted in the inset.

(4) The variation of single-particle level energies with distance from the surface. Shown are the affinity level and two ionization levels of Li obtained within a simple single-particle picture (see Ref[ 47]). The corresponding electron affinity and ionization energies of an isolated Li atom are given on the right hand side of the figure. Note that $z=0.0 \AA$ corresponds to the jellium edge, and the energy zero corresponds to the vacuum. The occupied levels of the metal are shown and the Fermi energy for the clean $\mathrm{Cu}$ surface lies $\Phi=4.59 \mathrm{eV}$ below the vacuum energy level. As the particle approaches the surface, the single-particle levels broaden into resonances (not shown).

(5) The measured relative yield of $\operatorname{Li}(2 \mathrm{p})$ (solid triangles) versus the work function shift, $\Delta \Phi$, induced by the deposition of $\mathrm{K}$, from the clean $\mathrm{Cu}(001)$ surface value of $4.59 \mathrm{eV}$. Here $\mathrm{Li}^{+}$is incident on $\mathrm{K} / \mathrm{Cu}(001)$ with $E_{o}=400 \mathrm{eV}$ and $\theta_{i}=65^{\circ}$. Noteworthy features in the data (solid triangles) include the peak occurring at $\Delta \Phi \approx 1.8 \mathrm{eV}$ and the large overall width of the peak. The solid line represents the prediction of the many-body model, normalized to the peak value of the measured yield.

(6) The charge fractions in the scattered flux, $\mathrm{P}^{+}$and $\mathrm{P}^{-}$, versus the work function shift, $\Delta \Phi$, induced by the deposition of Cs. Here the incident energy of $\mathrm{Li}^{+}$is $E_{o}=400 \mathrm{eV}$ and the scattering geometry is given by $\theta_{i}=65^{\circ}, \theta_{f}=64^{\circ}$. The lines show the predictions of the many-body model: solid for positive ion fraction, dashed for negative ion fraction.

(7) The peak energies $E / E_{0}$ in the measured energy spectra (open circles) are plotted as a function of incident energy $E_{0}$ and compared to the energies predicted by the classical trajectory simulation SAFARI (solid triangles and line). Energy loss through particle-hole pair formation is not included in the simulations, only energy transfer to the recoiling surface atoms. 


\section{REFERENCES}

[1] M. L. Yu and N. D. Lang, Phys. Rev. Lett. 50, 127 (1983).

[2] J. Los and J. J. C. Geerlings, Phys. Reports 190, 133 (1990).

[3] G. A. Kimmel, D. M. Goodstein, Z. H. Levine, and B. H. Cooper, Phys. Rev. B43, 9403 (1991).

[4] R. Brako and D. M. Newns, Rep. Prog. Phys. 52, 655 (1989).

[5] R. Brako and D. M. Newns, Surf. Sci. 108, 253 (1981).

[6] David C. Langreth and P. Nordlander, Phys. Rev. B43, 2541 (1991).

[7] For a review, see D. V. Averin and K. K. Likharev in Quantum Effects in Small Disordered Systems, edited by B. Al'tshuler, P. A. Lee, and R. A. Webb (Elsevier, New York, 1990), and references therein.

[8] C. M. Varma and Y. Yafet, Phys. Rev. B13, 2950 (1976); O. Gunnarsson and K. Schonhammer, Phys. Rev. B28, 4315 (1983).

[9] N. Andrei, Phys. Rev. Lett. 45, 379 (1980); N. Andrei and J. Lowenstein, Phys. Rev. Lett. 46, 356 (1981); P. B. Wiegmann, Zh. Eksp. Teor. Fiz. Pis'ma Red. 31, 392 (1980) [JETP Lett. 31, 364 (1981)].

[10] R. Brako and D. M. Newns, Solid State Comm. 55, 633 (1985).

[11] K. W. Sulston, A. T. Amos, and S. G. Davison, Phys. Rev. B37, 9121 (1988); A. T. Amos, K. W. Sulston, and S. G. Davison, "Theory of Resonant Charge Transfer in Atom-Surface Scattering", Advances in Chemical Physics, Vol. LXXVI, Molecular Surface Interactions, pp. 335 - 368, John Wiley \& Sons Ltd. (1989).

[12] K. W. Sulston, A. T. Amos, and S. G. Davison, Surf. Sci. 224, 543 (1991). 
[13] P. Nordlander and J. C. Tully, Phys. Rev. Lett. 61, 990 (1988); Surf. Sci. 211/212, 207 (1989); Phys. Rev. B42, 5564 (1990).

[14] P. Nordlander, Phys. Rev. B 46, 2584 (1992).

[15] H. Winter and R. Zimny, "Coherence in Grazing Ion-Surface Collisions," pp. 283 - 319 in Coherence in Atomic Collision Physics, Plenum Press, New York, NY 1988; R. Zimny, Surf. Sci. 233, 333 (1990).

[16] C. A. DiRubio, B. H. Cooper, G. A. Kimmel, and R. L. McEachern, Nucl. Inst. Meth. Phys. Res. B 64, 49 (1992); C. A. DiRubio, R. L. McEachern, J. G. McLean, and B. H. Cooper, in preparation.

[17] J. J. C. Geerlings, L. F. Tz. Kwakman, and J. Los, Surf. Sci. 184, 305 (1987).

[18] K. L. Sebastian, Phys. Rev. B31, 6976 (1985).

[19] H. Kasai and A. Okiji, Surf. Sci. 183, 147 (1987); H. Nakanishi, H. Kasai, and A. Okiji, Surf. Sci. 197, 515 (1988).

[20] Cyrus Umrigar, private communication.

[21] E. C. Goldberg, E. R. Gagliano, and M. C. G. Passeggi, Phys. Rev. B32, 4375 (1985).

[22] Peter Nordlander, Hongxiao Shao, and David C. Langreth, unpublished.

[23] A copy of the C-language program implementing these equations, "nstate.c," is available to interested parties. Please contact J. B. Marston via e-mail at: jbm@yollabolly.physics.brown.edu.

[24] A. J. Algra, E. V. Loenen, E. P. Th. M. Suurmeijer, and A. L. Boers, Radiat. Eff. 60, 173 (1982); B. Rasser, J. N. M. van Wunnik, and J. Los, Surf Sci. 118, 697 (1982).

[25] Nakanishi et al., Surf. Sci. 242, 410 (1991); Nakanishi et al., Surf. Sci. 216, 249 (1989).

[26] P. Nordlander, private communication. 
[27] A. G. Borisov, D. Teillet-Billy and J. P. Gauyacq, unpublished.

[28] D. L. Adler and B. H. Cooper, Rev. Sci. Instrum. 59, 137 (1988).

[29] R. L. McEachern, D. L. Adler, D. M. Goodstein, G. A. Kimmel, B. R. Litt, D. R. Peale, and B. H. Cooper, Rev. Sci. Instrum. 59, 2560 (1988).

[30] G. A. Kimmel and B. H. Cooper, to be published.

[31] E. R. Behringer, D. R. Andersson, B. H. Cooper, and J. B. Marston, to be published.

[32] H. Schall et al. Surf. Sci. 210, 163 (1989).

[33] P. O. Gartland, Phys. Norv. 6, 201 (1972; P. O. Gartland, S. Berge, and B. J. Slagsvold, Phys. Norv. 7, 39 (1973).

[34] J. J. C. Geerlings, R. Rodink, J. Los, and J. P. Gauyacq, Surf. Sci. 186, 15 (1987).

[35] H. Brenten et al. Surf. Sci. 243, 309 (1991).

[36] M. J. Ashwin and D. P. Woodruff, Surf. Sci. 244, 247 (1991).

[37] H. Brenten et al. Nucl. Inst. Meth. B58, 328 (1991).

[38] D. M. Goodstein, S. A. Langer, and B. H. Cooper, J. Vac. Sci. Technol. A 6, 703 (1988).

[39] Craig Keller and B. H. Cooper, unpublished.

[40] C. C. Hsu, H. Bu, A. Bousetta, J. W. Rabalais, and P. Nordlander, Phys. Rev. Lett. 69, 188 (1992).

[41] Y. Bu, E. F. Greene, and D. K. Stewart, J. Chem. Phys. 92, 3899 (1990).

[42] J. J. C. Geerlings, R. Rodink, J. Los, and J. P. Gauyacq, Surf. Sci. 181, L177 (1987).

[43] Tony Fónden and Andre Zwartkruis, Surf. Sci. 269/270, 601 (1992); N. Lorente and R. Monreal, unpublished; R. Zimny, Surf. Sci. 255, 135 (1991) and unpublished. 
[44] H. Schall, H. Brenten, K. H. Knorr, and V. Kempter, Z. Phys. D16, 161 (1990).

[45] C. Kane and M. Fisher, Phys. Rev. Lett. 68, 1220 (1992) and preprint.

[46] Alan T. Dorsey, Karsten W. Jacobsen, Zachary H. Levine, and John W. Wilkins, Phys. Rev. B40, 3417 (1989).

[47] G. A. Kimmel and B. H. Cooper, to be published. 